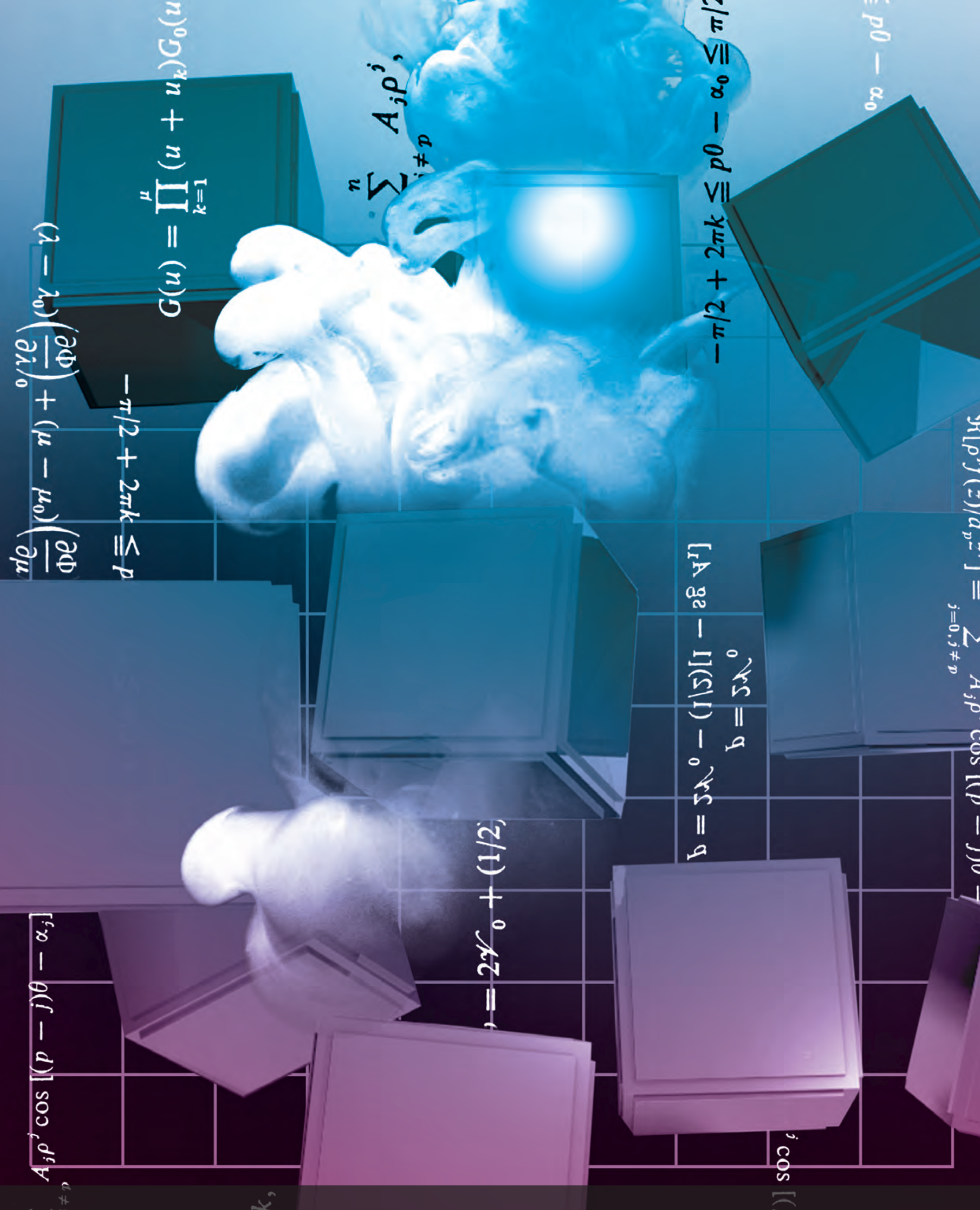




\section{LA CAJA DE POLINOMIOS Y EL MÉTODO TRADICIONAL: DOS ALTERNATIVAS DIDÁCTICAS PARA LA ENSEÑANZA DE LA SUMA Y LA RESTA DE POLINOMIOS}

\author{
The polynomial's box and the traditional method: Two didactic alternatives in the teaching of \\ addition and subtraction of polynomials
}

A caixa de polinômios e o método tradicional: Duas alternativas didáticas para o ensino da soma e a resta de polinômios
RECIBIDO:4DE ABRIL DE 2017,

José Martin Villarroel Solis

martinvilla15@gmail.com

Msc. en Enseñanza de la Ciencias

Universidad Minuto de Dios

\section{es}

\section{RESUMEN}

En este trabajo se pretendió comparar el nivel de aprendizaje alcanzado por estudiantes de grado octavo de la institución educativa (I.E.) María Cano (Medellín-Antioquia), en los temas de suma y resta de polinomios cuando este tema es abordado con el método tradicional y usando la herramienta didáctica la caja de polinomios. Se toman cuatro grupos distintos de grado octavo y se distribuyen en dos grupos. En dos de ellos se trabaja por medio de la herramienta didáctica la caja de polinomios, fundamentando el trabajo en la teoría cognitiva propuesta por David Ausubel, denominada aprendizaje significativo, y en los otros dos aplicando metodología tradicional, que consiste en explicar-ejemplificar-ejercitar, que para efectos de este trabajo se ha denominado (EEE). Finalmente, se contrastaron los resultados cualitativa y cuantitativamente; Los promedios de notas de los grupos no varían, con respecto a los promedios históricos, la diferencia entre promedios es relativamente pequeña. Si bien las notas no presentan mejoría, la dispersión se reduce significativamente, la utilización de material didáctico en las aulas de clase no garantiza que los estudiantes alcancen el objetivo de aprendizaje, pero influye positivamente en aspectos relevantes en el proceso de enseñanza-aprendizaje, tales como: organización, interés y motivación por el conocimiento, participación activa, interacción, entre otros.

PALABRAS CLAVE: aprendizaje significativo, caja de polinomios, adición y sustracción de polinomios..

\section{EVALUADO:25 DE JUIO DE 2017}

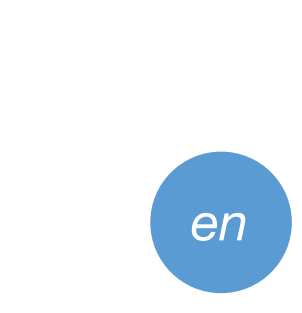

\section{ABSTRACT}

The aim of this study was to compare the level of learning achieved by eighth-grade students of the María Cano (MedellinAntioquia) educational institution (E.I), in the subject of addition and subtraction of polynomials when this subject is approached with the traditional method and using the polynomial's box didactic tool. Four different eighth-grade groups are taken and distributed into two groups. In two of them, the polynomial's box didactic tool is used, based in the meaningful learning, cognitive theory proposed by David Ausubel. In the other two groups the traditional methodology was applied, which consists in explain-exemplify-exercise which, for the purposes of this study, has been called (EEE). Finally, the results were qualitatively and quantitatively compared. Group average grades do not vary from historical averages. The difference between averages is relatively small. Although grades do not show improvement, the dispersion is significantly reduced. Conclusions. The use of didactic material in classrooms does not guarantee that students will reach the learning goal, but positively influences relevant aspects in the teaching-learning process, such as: organization, interest and motivation for knowledge, active participation, and interaction, among others..

KEYWORDS: significant learning, polynomial's box, addition and subtraction of polynomials.

\section{ACEPTADO: 8DE AGOSTO DE 2017}

Jhoana Patricia Romero-Leiton johana.romeroleiton@udea.edu.co

Doctora en Matemáticas Universidad de Antioquia

\section{por}

\section{RESUMO}

Em este trabalho pretendeu-se comparar o nível de aprendizado conseguido por estudantes da oitava série da instituição educativa (I.E.) Mária Cano (Medellín-Antioquia), nos temas de soma e resta de polinômios quando este tema é abordado com o método tradicional e usando a ferramenta didática a caixa de polinômios. Se tomam quatro grupos diferentes da oitava série e distribuem-se em dois grupos. Em dois deles trabalha-se através da ferramenta didática a caixa de polinômios, fundamentando o trabalho na teoria cognitiva proposta por David Ausubel, denominada aprendizado significativo, e nos outros dois aplicando metodologia tradicional, que consiste em explicar-exemplificar-exercitar, que para efeitos deste trabalho tem-se denominado (EEE). Finalmente, se contrastaram os resultados qualitativa e quantitativamente. As médias de notas dos grupos não variam, a respeito às médias históricas. A diferença entre médias é relativamente pequena. Embora as notas não apresentam melhoria, a dispersão se reduz significativamente. Conclusões. A utilização de material didático nas salas de aula não garante que os estudantes consigam $o$ objetivo de aprendizado, mas influi positivamente em aspectos relevantes no processo de ensino-aprendizado, tais como: organização, interesse e motivação pelo conhecimento, participação ativa, interação, entre outros.

PALAVRAS CHAVE: aprendizado significativo, caixa de polinômios, adição e subtração de polinômios. 


\section{INTRODUCCIÓN}

Al ser la didáctica "la ciencia de la educación que estudia $e$ interviene en el proceso de enseñanza-aprendizaje con el fin de conseguir la formación intelectual del educando" (Mallat, 2011, p.5), demanda de sus profesionales producir y/o utilizar herramientas para facilitar el proceso de enseñanza-aprendizaje. Un número considerable de profesores han dado un paso más en el proceso, documentando su trabajo, por lo que actualmente se cuenta con gran variedad de textos que describen experiencias que se han aplicado para enseñar. Siguiendo el trabajo de Vinholi (2011), quien utiliza la teoría de aprendizaje significativo de David Ausubel para enseñar conceptos de botánica en las escuelas de Brasil, los autores han implementado estrategias metodológicas en el aula de acuerdo con las características del material didáctico caja de polinomios. En el campo de la didáctica de las matemáticas se cuenta con algunos registros como el de Urbano (2011) y Valbuena et al. (2015), quienes trabajaron enseñanza de matemáticas por medio del computador en la escuela.

Las universidades que ofrecen carreras en formación de maestros, particularmente en el área de matemáticas, y aun las que no, cuentan con aulas-taller especializadas en la creación y experimentación de material didáctico para la enseñanza de los diferentes conceptos matemáticos, al punto que se podría encontrar material propio para cada tema; esto, sumado a la dinámica de aprendizaje de los estudiantes contemporáneos, ha conllevado a que sea una necesidad utilizar herramientas didácticas para enseñar matemáticas en el aula.

La caja de polinomios es un material didáctico que fue creado para enseñar matemáticas. Su creador es el magíster Óscar Fernando Soto, profesor de la Universidad de Nariño, con el fin de brindar a los maestros de la región una alternativa didáctica para enseñar operaciones básicas con polinomios, así como también el proceso de factorización. Este material ha sido aplicado en ciertos contextos educativos; experiencias que han quedado documentadas. Soto, Gómez y Mosquera (2005), dan a conocer a la comunidad académica el material didáctico que denominaron la caja de polinomios, sometiendo a revisión un artículo con el mismo nombre, el cual fue publicado en el volumen 13 de la Revista de la Escuela Regional de Matemáticas (ERM). Soto, Lozano y Naranjo (2009), utilizaron este material para enseñar operaciones básicas con polinomios a personas en condición de discapacidad auditiva. Por su parte, Villarroel (2014), adaptó este material para generar una propuesta didáctica para enseñar, además de operaciones básicas con polinomios, el proceso de factorización, fundamentando teóricamente la propuesta de caja polinomios con la teoría cognitiva de aprendizaje significativo de David Ausubel. Este trabajo se convirtió finalmente en su tesis de maestría.

Hablar de aprendizaje significativo necesariamente remite al trabajo de Marco Moreira, quien en el Encuentro internacional sobre aprendizaje significativo expone, básicamente, que las personas aprenden a partir de lo que ya saben, principio que originó, en su momento, la propuesta de Ausubel. Pero, además, incorpora a este principio la idea de abandonar la narrativa en el aula de clase, a lo que denominó aprendizaje significativo crítico, Moreira (1997).

La intención de este trabajo es tomar dos de los cuatro grupos de grado octavo de la Institución Educativa María de los Ángeles Cano Márquez (Medellín-Antioquia), y aplicar la propuesta para la enseñanza de la suma y la resta de polinomios que surge al asociar la propuesta de la caja de polinomios con la teoría cognitiva de aprendizaje significativo propuesto por David Ausubel, al tiempo que en los dos grupos restantes del mismo grado se trabaja con el método convencional con que generalmente se enseña, al que se ha denominado para efectos del presente documento como el método EEE (explicar, ejemplificar, ejercitar). Pero además, y principalmente, realizar el correspondiente análisis de los resultados obtenidos después de aplicar las dos metodologías, desde las perspectivas cuantitativa y cualitativa. La primera, aplicando algunos conceptos de estadística descriptiva, y la segunda, estableciendo un cuadro que compara las características de aspectos que los maestros consideran fundamentales en el desarrollo de una clase. Con ello, se divulga la existencia del material didáctico y se dan a conocer sus características fundamentales, proporcionando a los maestros la información que se requiere cuando se trata de elegir la herramienta didáctica para utilizar en el aula de clase.

\section{Metodología}

Los grupos considerados en el trabajo fueron 8.1, 8.2, 8.3 y 8.4 de la I.E. María Cano del municipio de
| Panorama | pp. 20-32 
José Martin

Villarroel Solis

Jhoana Patricia

Romero-Leiton

\section{DISEÑO DE MATERLAL DIDÁCTICO}

En esta etapa se seleccionaron las guías de aprendizaje que se aplicaron con los grupos CP, las cuales fueron diseñadas por uno de los autores del presente documento, como parte de la tesis de grado para optar al título de magíster en enseñanza de las ciencias. Se debe destacar que cada estudiante de los grupos $\mathrm{CP}$ aportó la materia prima para construir las cajas de polinomios, las cuales fueron elaboradas por un grupo de estudiantes de octavo en compañía del docente.

\section{ORGANIZACIÓN PREVIA}

Según la teoría de Ausubel, es indispensable que quien aprende tenga los conocimientos necesarios para aprender la nueva información. Ausubel llama a estos pre-conceptos o pre-requisitos, subsumidores (Moreira, 1997). Por ello, se trató al máximo de garantizar que los cuatro grupos trabajaran y aprendieran los siguientes subsumidores: operaciones básicas con números reales, potenciación, reducción de términos semejantes. Aclarando que se enfatizó en la parte técnica, dado que estas operaciones son procesos que se aplican en las operaciones básicas con polinomios.

Para el trabajo con la caja de polinomios hubo necesidad de dedicar dos sesiones extra con los grupos $\mathrm{CP}$, con la finalidad de explicar el funcionamiento del material, pues era importante que todos los estudiantes adquirieran pericia en los procesos de representación de polino-

Panorama

pp. 20-32

Volumen 10

Número 20

Enero-Junio

Tradicionalmente, y por razones específicamente matemáticas, se trabajaron las operaciones con polinomios de manera similar como se trabajan las operaciones básicas con números reales, esto es, en el siguiente orden:

\section{Método EEE}

adición y luego sustracción (entendida como una suma con un inverso aditivo).

AdICIÓN

Explicar: básicamente se explicó en el tablero que para sumar polinomios se construye un nuevo polinomio uniendo los polinomios, sumando mediante el signo más “+”, y posteriormente reduciendo términos semejantes si los hubiera.

Ejemplificar: en esta etapa del método se mostraron varios ejemplos, tratando de abordar al máximo los posibles casos que se pudieran presentar. Entre ellos se planteó:

Sean

$P=3 a+6 b+2 c$ y $Q=-5 a+2 b+c$

Con ellos determinar $P+Q$

$P+Q=3 a+6 b+2 c+(-5 a+2 b+c)=3 a+6 b+2 c-5 a+2 b+c$

$$
=-2 a+8 b+3 c
$$

Además, se les indica un proceso alternativo, ubicando uno de los polinomios sumando en una línea, y el otro sumando en la línea inferior, garantizando que cada término del segundo sumando quede debajo de su semejante en el primer polinomio y posteriormente reduciendo los términos semejantes.

Sean $P=3 a+6 b+2 c$ y $Q=-5 a+2 b+c$

Con ellos determinar $P+Q$

$$
\begin{gathered}
P=3 a+6 b+2 c \\
Q=-5 a+2 b+c \\
P+Q=-2 a+8 b+3 c
\end{gathered}
$$

Ejercitar: se proponen ejercicios de menor a mayor nivel de complejidad. Primero, adición de monomios, adición de binomios y finalmente, adición de polinomios. 
teoría de aprendizaje significativo propuesta por David Ausubel.

Explicar: básicamente, se explica que, si se tienen dos polinomios $P$ y $Q$, con ellos se pueden determinar dos nuevos polinomios: $P-Q$ y $Q-P$. Además, que el polinomio $P-Q$ se interpreta como la suma entre el polinomio $P$ con el polinomio opuesto al polinomio $Q$, es decir $P-Q=P+(-Q)$. Explicando que para construir el polinomio opuesto a un polinomio dado se cambian los signos a todos los términos del polinomio inicial.

Otro aspecto relevante que se explica es la relación que hay entre $P-Q$ y $Q-P$, pues justamente una diferencia corresponde al opuesto de la otra, es decir, $P-Q=-(Q$ $-P) \circ Q-P=-(P-Q)$

Ejemplificar: consideremos los polinomios

$P=-2 x^{2}+3 x y-2 z y Q=z+5 x^{2}-6 x$

Determinar $P-Q$

Como se mencionó $P-Q=P+(-Q)$ por lo que hay que sumar los polinomios $P y-Q$, lo cual requiere construir el polinomio opuesto de $Q$.

$$
\begin{aligned}
-Q & =-\left(z+5 x^{2}-6 x y\right) \\
& =-z-5 x^{2}+6 x y
\end{aligned}
$$

Ahora, se efectúa la suma, para ello se utiliza el método que cada estudiante elija para sumar. Tomemos el proceso que denominamos alternativo.

\begin{tabular}{c}
$P=-2 x^{2}+3 x y-2 z$ \\
$-Q=-5 x^{2}+6 x y-z$ \\
\hline$P-Q=-7 x^{2}+9 x y-3 z$
\end{tabular}

Ejercitar: al igual que en la adición, se proponen ejercicios de menor a mayor nivel de complejidad.

\section{Método caja de polinomios}

A continuación, se presenta el análisis de la experiencia de aplicar las guías de aprendizaje, cuya finalidad fue propiciar el aprendizaje de las operaciones de adición y sustracción de polinomios, tomando como referencia la
CONSTRUCCIÓN DEL MATERIAL

La caja de polinomios es una herramienta didáctica que se puede utilizar para la enseñanza de diferentes conceptos de las matemáticas escolares, lo cual invitaría a pensar que la construcción corresponde a fabricantes especialistas únicamente; sin embargo, para llevar a cabo esta investigación fueron los estudiantes quienes aportaron los materiales y, lo más importante, quienes construyeron sus propias cajas de polinomios, bajo la supervisión del docente del área.

\section{Fundamentación teórico-matemática de la caja de polinomios}

La caja de polinomios es una herramienta que tiene fundamentos matemáticos en todos los aspectos que la definen: fichas, tablero y funcionamiento.

FICHAS

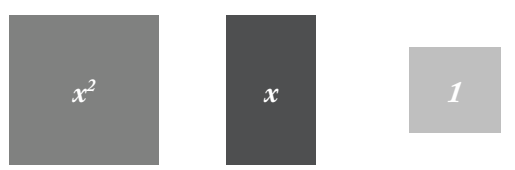

Figura 1: Fichas caja de polinomios

Las primeras fichas, las cuales están dadas en la Figura 1 , son de tres tipos diferentes: cuadrado grande, rectángulo y cuadrado pequeño. Para el cuadrado grande (color azul), se define una distancia $x$ que se asignará como la medida de los lados, por lo cual el área del cuadrado será $(\mathrm{x})(\mathrm{x})=\mathrm{x}^{2}$. Para el caso del rectángulo (color verde), se toma la distancia $x$ para una de las dimensiones y se define una distancia unidad (1) para la otra dimensión, con lo que se tiene un rectángulo de dimensiones x y 1 , por cuanto el área será $(\mathrm{x})(1)=x$. Finalmente, se construye un cuadrado pequeño (color amarillo), de lados correspondientes a la unidad, por lo que el área será (1) $(1)=1$. Las fichas se relacionan justamente porque las dimensiones del rectángulo coinciden exactamente con las dimensiones de las otras dos, lo cual permite que las tres fichas se unan por lados correspondientes de manera precisa, formando una figura como muestra la Figura 2
Panorama | pp. 20-32

Volumen 10

| Número 20

| Enero-Junio

2016 


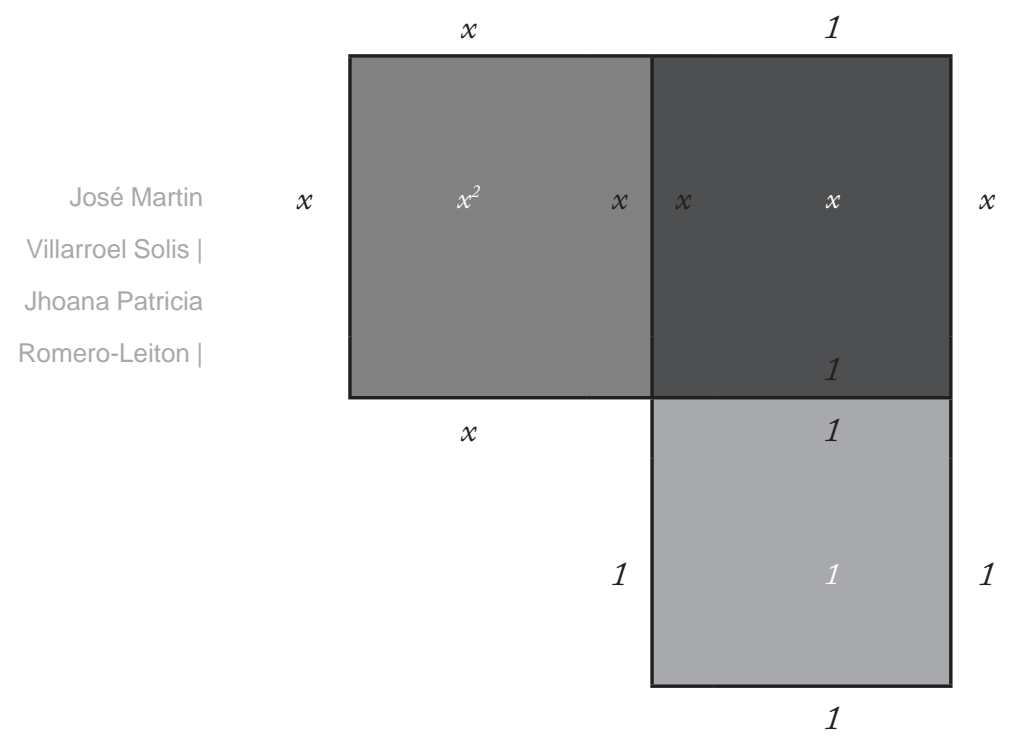

Figura 2: Relación entre dimensiones de las fichas

TABLERO

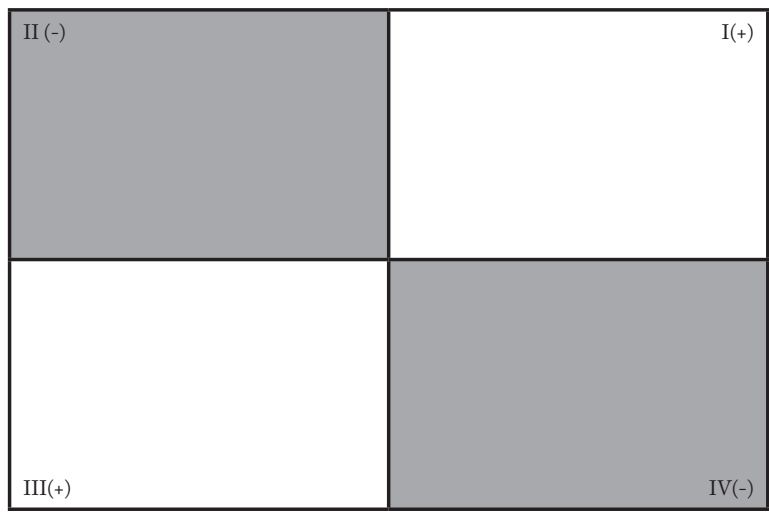

Figura 3:Tablero

El tablero corresponde a una región rectangular que emula al plano cartesiano, por lo cual está dividido en cuatro subregiones por dos segmentos de recta perpendiculares, que unen los puntos medios de los lados.

Panorama

pp. 20-32

Volumen 10

Número 20

Enero-Junio

$2017 \mid$

Como se observa en la Figura 3, se nombra cada una de las subregiones similar a los cuadrantes del plano, así mismo se le asignan los signos. El cuadrante superior derecho será el primer cuadrante y tendrá signo positivo, el superior izquierdo será el segundo cuadrante con signo negativo, el inferior izquierdo será el tercer cuadrante con signo positivo y el inferior derecho será el cuarto cuadrante y tendrá signo negativo. Esta asignación de los signos tiene el mismo fundamento de los signos de los cuadrantes del plano, es decir, en el tablero

24 | de la caja de polinomios también consideraremos ejes coordenados. La intersección de los segmentos será el origen, el segmento comprendido entre el origen y el punto medio de la altura derecha del tablero representará medidas positivas y el de la izquierda, medidas negativas. De igual manera, el segmento que va del origen al punto medio de la base representará medidas negativas y el que va del origen a la base superior representará medidas positivas.

\section{Relación fichas-tablero}

Para ubicar las fichas en el tablero es importante tener presente dos aspectos fundamentales. PRIMERO: el área de todas las fichas debe estar totalmente contenida en una de las regiones del plano, pues de la ubicación de la ficha en las diferentes regiones depende el signo que esta adquiere. SEGUNDO: si uno o los dos lados de una ficha se ubican sobre los ejes, estos adquirirán el signo de dicho eje.
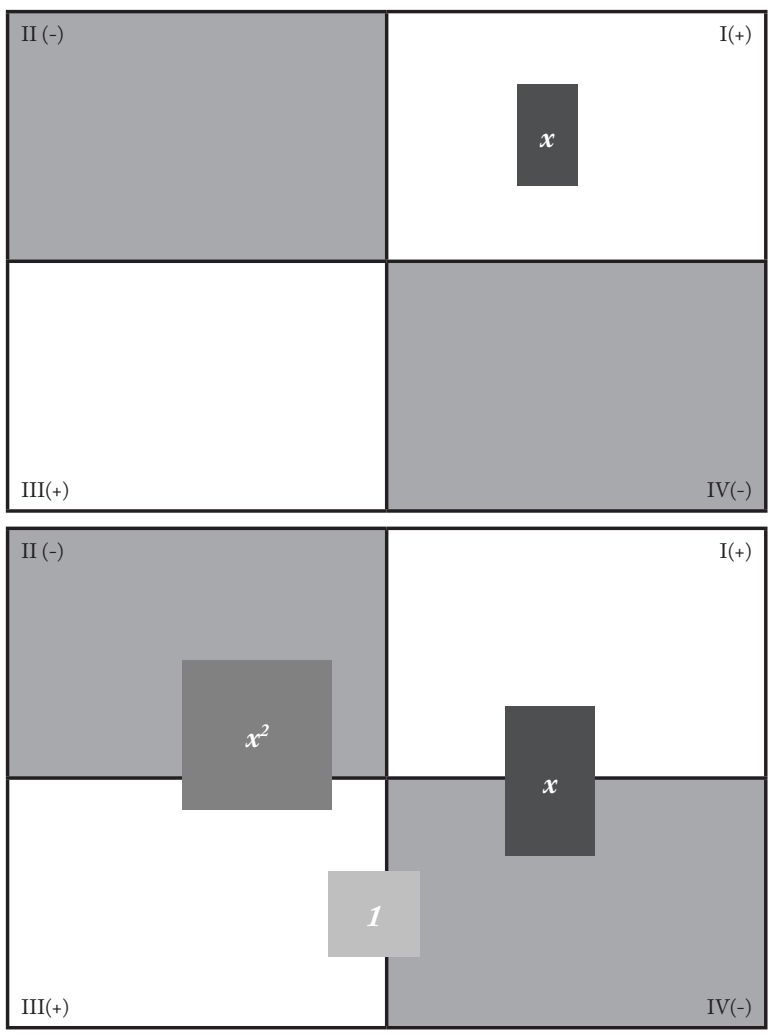

Figura 4: Ubicación correcta de las fichas

La Figura 4 (derecha) muestra la forma correcta de colocar una ficha en el tablero, por cuanto que el total de la ficha está contenida en el primer cuadrante. Por su parte, la Figura 4 (izquierda) muestra tres fichas 
colocadas de manera incorrecta porque, en los tres casos, el área de las fichas esta sobre dos cuadrantes.

\section{Escritura de polinomios en el tablero para adición y sustracción}

Se trabaja como polinomios de la forma $P(x)=a x^{2}+b x+c$ con $a, b$, cen $Z$, es decir, polinomios de segundo grado en una sola variable con coeficientes enteros. La escritura de polinomios se diferencia según la operación que se pretenda realizar en el tablero.

Los signos de los cuadrantes permiten representar los signos de los coeficientes, mientras que la cantidad de fichas dispuesta en el tablero representa justamente el valor absoluto del coeficiente, es así, por ejemplo, el polinomio $P(x)=-2 x^{2}+3 x-1$ tiene diversas formas de representarse en el tablero. Una de ellas sería ubicando dos fichas ' $\mathrm{x}^{2}$ 'en el segundo cuadrante; tres fichas ' $\mathrm{x}$ ' en el primer cuadrante y finalmente una ficha ' 1 ' en el cuarto cuadrante. La Figura 5 muestra dos disposiciones diferentes de fichas que representan al polinomio $P$.
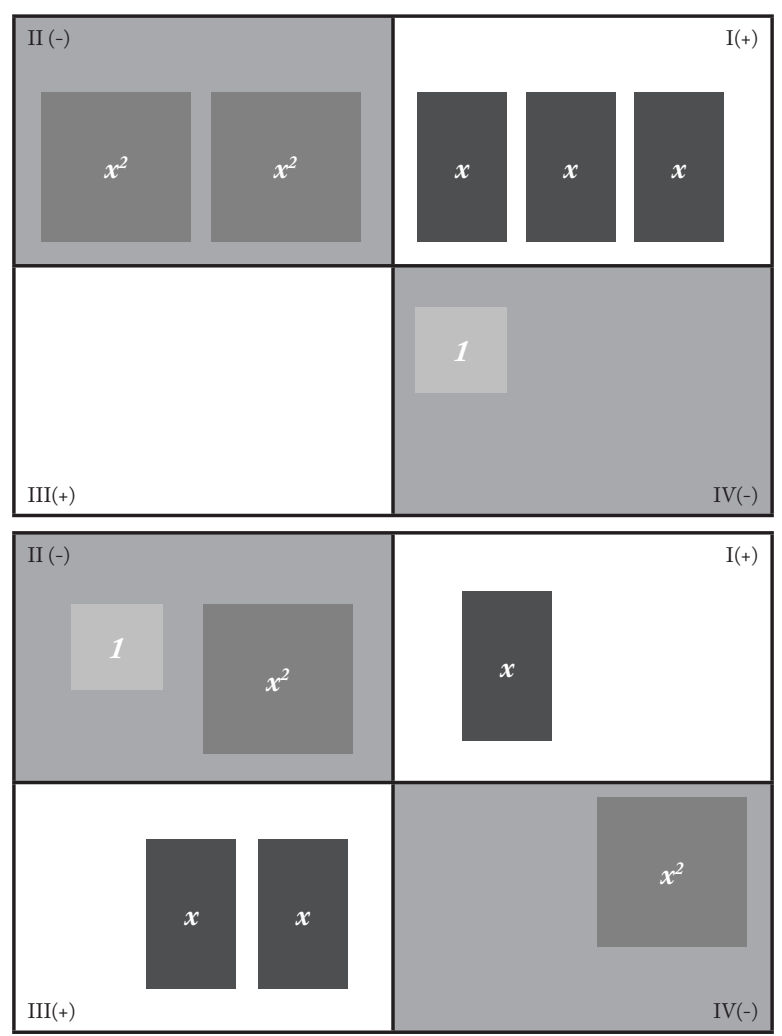

Figura 5: Representación equivalente adición.

\section{Ejecución de las operaciones básicas con la caja de polinomios}

Ceros

Un concepto fundamental que se utilizará se denomina cero, que matemáticamente hace referencia a la propiedad invertible de la adición de números reales. La caja de polinomios permite representar por medio de las fichas esta propiedad tan esencial de la operación de adición de una manera muy fácil de entender, pues dos fichas de igual peso algebraico ubicadas en cuadrantes de signos opuestos son opuestas, por lo cual su suma será un cero, y por esto es posible representar en el tablero un cero de múltiples formas.

ADICIÓN

Al ser la adición una operación binaria, es necesario representar dos polinomios en el tablero, pero también es fundamental que se identifique claramente las fichas que corresponden a cada uno de los polinomios. Para ello se recomienda que se utilicen el primer y cuarto cuadrante para representar uno de los sumandos, y el segundo y tercer cuadrante para representar el otro sumando, esto hará que no se confundan las fichas de cada polinomio.

Una vez que se tengan representados los dos polinomios sumandos en el tablero, simplemente se procede a aplicar la propiedad invertible de la adición de reales, es decir, a buscar la mayor cantidad de ceros posibles. Dependiendo de los signos de cada término de los polinomios, puede presentarse el caso de tener fichas del mismo tipo en cuadrantes del mismo signo, en ese caso las fichas no forman ceros, por lo cual se deben sumar, lo que implica que se trasladen las fichas a un mismo cuadrante. Finalmente, se hace lectura del polinomio que queda representado en el tablero después de levantar las fichas que constituyen los ceros. Dicho polinomio es la suma de los dos polinomios que se pretendía en principio sumar.

Sean $P(x)=-x^{2}+3 x-1$ y $Q(x)=-2 x^{2}+x+3$

La Figura 6 muestra la representación de los polinomios en el tablero. Observe que las fichas $\mathrm{x}^{2} \mathrm{y} x$ de los dos polinomios están ubicadas en cuadrantes del mismo
| Panorama | pp. 20-32 | Volumen 10 
José Martin Villarroel Solis | Jhoana Patricia Romero-Leiton | signo, respectivamente, esto significa que no forman ceros y que deben ser sumadas, para lo cual simplemente se las une. Con las fichas 1 , si se determina un cero, esto implica que se debe levantar del tablero una ficha 1 de cada polinomio. Finalmente, se hace lectura del polinomio que queda representado en el tablero, siendo este la suma entre $P$ y $Q$. La Figura 6 muestra la suma de los polinomios representada en el tablero, siendo $\mathrm{P}(x)+\mathrm{Q}(\mathrm{x})=-3 \mathrm{x}^{2}+4 \mathrm{x}+2$.
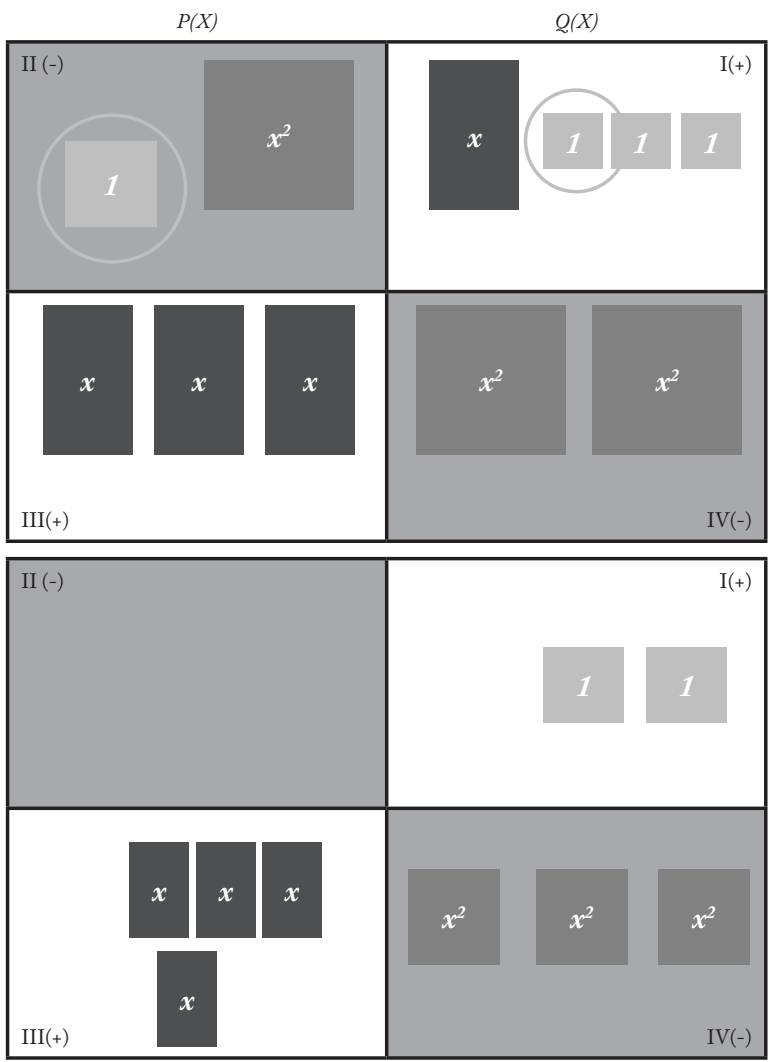

Figura 6: Ubicación de los sumandos

SUSTRACCIÓN

Al definirse la operación de adición sobre el conjunto de los polinomios, esta cumple con la propiedad invertible, es decir, dado un polinomio cualquiera, existe otro polinomio tal que la suma de ellos es el polinomio cero, en ese caso los polinomios en cuestión son opuestos mutuamente.

Si $P$ y $Q$ son polinomios y $P+Q=Q+P=0$ entonces $P=-Q$ o

$Q=-P$

Esta propiedad elemental, pero de gran importancia,

26 | permite que, dado un polinomio, se construya a partir de él su opuesto. En la práctica esto se hace cambiando los signos de todos los coeficientes del polinomio. Entonces,

Si $P(x)=3 x^{2}-5 x+5$ entonces,

$$
\begin{aligned}
-\mathrm{P}(x) & =-\left(3 x^{2}-5 x+5\right) \\
& =-3 x^{2}+5 x-5
\end{aligned}
$$

\section{Representación del polinomio opuesto en el tablero}

Como se mencionó anteriormente, la sustracción es una adición que toma como sumandos un polinomio y el opuesto de otro polinomio; en ese sentido, todo lo que se definió para la adición de polinomios se cumplirá para la sustracción. El elemento diferencial entre los dos procedimientos está en que la operación $P-Q$, se entiende como $P+(-Q)$, es decir, la suma entre el polinomio $P$ con el opuesto del polinomio $Q$.

Al representar en el tablero, sigue vigente el hecho de tomar dos cuadrantes para cada polinomio, como se estableció para la adición, pero, una vez ubicado el polinomio sustraendo $Q$, se construye su opuesto cambiando las fichas de cuadrante, es decir, si se ubican las fichas del polinomio sustraendo en el I y IV cuadrante, entonces la construcción del opuesto consiste en trasladar las fichas del I cuadrante al IV cuadrante; de igual manera, las del IV cuadrante al I cuadrante. Matemáticamente, eso significa cambiar el signo de los términos del polinomio en cuestión, como lo muestra la Figura 7.

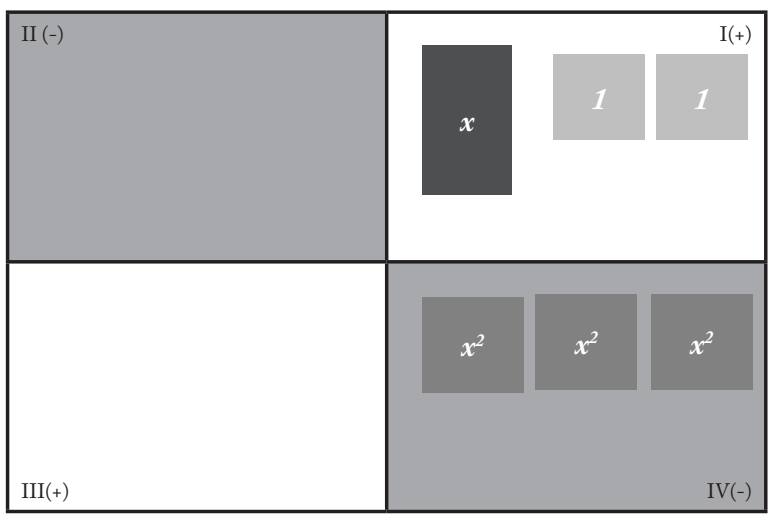




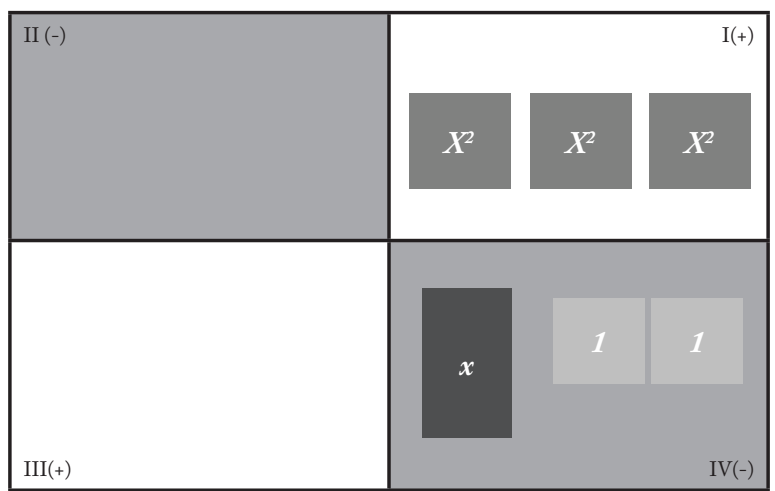

Figura 7: Polinomio P y polinomio opuesto de P.

PROCESO PARA SUSTRAER

Una vez ubicados los polinomios minuendo y sustraendo, y construido el polinomio opuesto del sustraendo, el proceso ha avanzado en gran medida, pues solamente hace falta identificar la mayor cantidad de ceros entre las fichas del polinomio minuendo y opuesto del sustraendo, que al igual que en la adición deben ser del mismo tipo, o trasladar las fichas del mismo tipo que estén en cuadrantes de igual signo. Por último, realizar lectura del polinomio que queda representado en el tablero, que será la diferencia buscada.

A continuación, como muestra la Figura 8, se identifican todos los ceros que se forman entre las fichas de $P$ con las de $-Q$, y retiran del tablero, se trasladan de cuadrante las fichas que tienen el mismo signo $y$, finalmente, se hace lectura del polinomio que queda representado en el tablero, por cuanto: $\mathrm{P}(\mathrm{x})-\mathrm{Q}(\mathrm{x})=-\mathrm{x}^{2}-\mathrm{x}$ -4 .

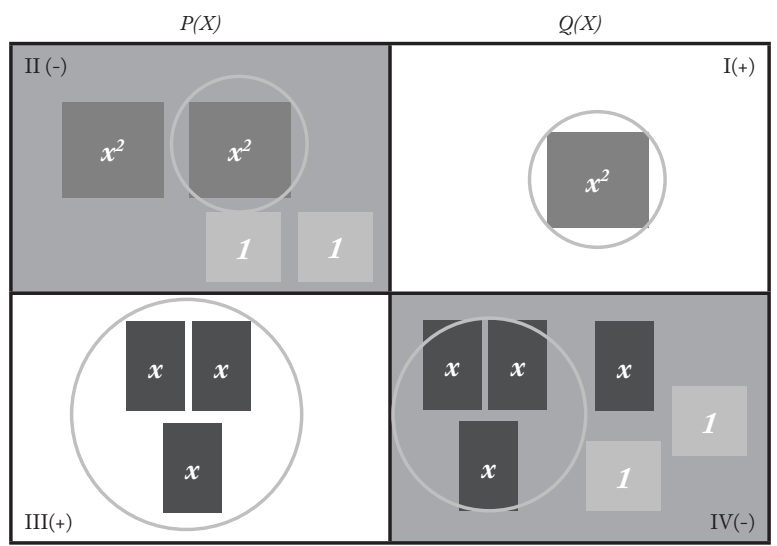

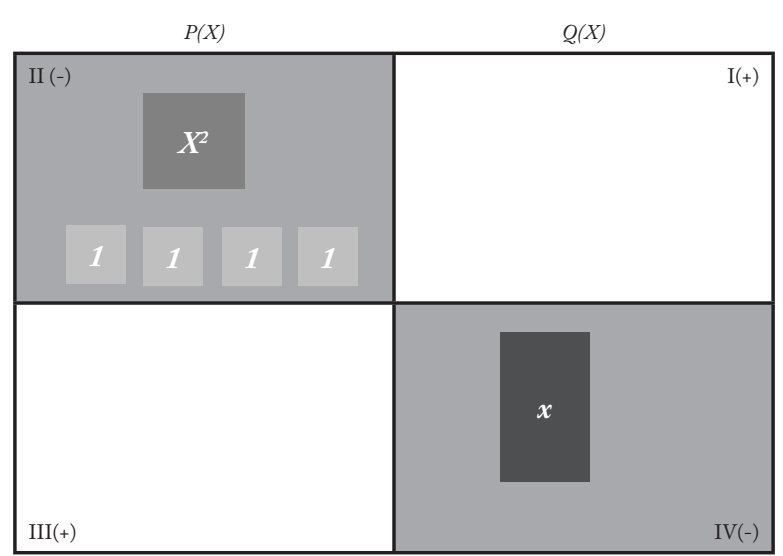

Figura 8: Identificación de ceros y resultado de la sustracción

\section{Caja de Polinomios y aprendizaje significativo}

\section{Relación: material-estructura cognitiva}

Ausubel propone en su teoría de aprendizaje significativo, entre otras cosas, que el material presentado a quien aprende debe ser potencialmente significativo, es decir, que pre-exista una relación entre el material y algún concepto de los que ya componen la estructura cognitiva del estudiante (Moreira, 1997). Los elementos que conforman la caja de polinomios, con sus características y propiedades, son altamente vinculantes con el conocimiento que un estudiante de grado octavo debe tener incorporado en su estructura cognitiva, pues son conceptos que se han trabajado desde el nivel de básica primaria.

Las diferentes fichas que componen la caja de polinomios corresponden a cuadrados y rectángulos, figuras geométricas que todos los estudiantes identifican desde muy temprana edad. $\mathrm{El}$ área de cada ficha representa la parte literal del término. Ahora bien, al ubicar las diferentes fichas en el tablero, adquieren un signo, pues si se ubica una ficha en los cuadrantes I o III, la ficha representará un término con signo positivo; de similar manera, si se ubica en los cuadrantes II y IV, representará un término negativo. Por su parte, los coeficientes de los términos se ven representados en la cantidad de unidades de cada ficha. Hay que precisar que la caja de polinomios permite la representación de términos con coeficientes enteros, siendo la finalidad que se lleve a cabo un proceso de abstracción, que permita sumar o restar polinomios, con cualquier tipo de coeficiente, sin necesidad de utilizar la herramienta.
La caja de polinomios y el método tradicional: Dos alternativas didácticas para la enseñanza de la suma y la resta de polinomios
Panorama | pp. 20-32 Volumen 10 
José Martin

Villarroel Solis |

Jhoana Patricia

Romero-Leiton |

\section{Subsumidores}

Ausubel plantea que previo a trabajar el nuevo conocimiento debe existir una etapa en la cual se trabajen los conocimientos requeridos, los cuales, como se mencionó anteriormente, se denominan subsumidores (Moreira, 1997). Para el caso de los procesos de suma y resta de polinomios con caja de polinomios, los subsumidores son: manejo del plano cartesiano, perímetro y área de rectángulos y cuadrados.

La teoría ausubeliana propone que los subsumidores de cada nuevo tema se deben trabajar de la manera más abstracta posible, buscando que quien aprende alcance un nivel máximo de apropiación de ellos, con el fin de poder utilizarlos y aplicarlos cuando se requiera, sin dificultad alguna (Moreira, 1997). Fue por ello que, previo al trabajo con caja de polinomios, se trabajaron durante dos sesiones de clase los subsumidores mencionados.

\section{Subsunción}

Se entiende por subsunción el proceso mediante el cual la nueva información se ancla a un concepto ya establecido en la estructura cognitiva de quien está aprendiendo, para que finalmente se incorpore a ella (Moreira, 1997). En el trabajo con la caja de polinomios, los conceptos que Ausubel denomina ancla son: el concepto de área de cuadrados y rectángulos, suma de cantidades del mismo signo, de signos diferentes y opuestos.

Panorama

pp. 20-32

Volumen 10

Número 20 |

Enero-Junio

Es importante mencionar que el proceso de subsunción es, finalmente, el objetivo de quien pretende enseñar, pero en cada caso este se lleva a cabo de diferentes formas. En la experiencia con la caja de polinomios no fue diferente, pues se realizó en diferentes etapas, que vistas integralmente dan como resultado que el estudiante aprendió los conceptos de suma y resta de polinomios, lo que se traduce en que el estudiante abstrajo e incorporó a su estructura cognitiva el proceso para sumar y restar cualquier tipo de polinomios que se le presente, sin necesidad de utilizar o representarlos mediante la caja de polinomios.

\section{Trabajo en el aula}

Como se mencionó, con los grupos $\mathrm{CP}$ se trabajaron dos sesiones extra para aplicar la guía de aprendizaje correspondiente a los fundamentos de la caja de polinomios. Con ello se pretendió que los estudiantes, inicialmente, tuvieran un primer acercamiento al material, lo manipularan, y por qué no, jugaran con él, pues el fundamento de la caja de polinomios está en el juego (Soto et al., 2005). Seguido a esto, se explica el funcionamiento, características y fundamento de cada uno de los elementos que componen el material. En esta etapa del proceso se trató al máximo de no omitir detalle alguno, pues entre más elementos y conceptos resultaran vinculantes para el estudiante, existe más posibilidad de anclar el nuevo conocimiento.

Una vez que los grupos conocen el funcionamiento de la caja de polinomios de forma adecuada, entendiendo esto como que saben ubicar correctamente las fichas en los diferentes cuadrantes, representar cualquier término de diversas maneras, y en general, escribir cualquier polinomio que la herramienta lo permita, de varias formas y hacer lectura correcta de un polinomio representado en el tablero, se procede a aplicar la guía de suma y resta de polinomios. Esta guía, al igual que la anterior, se compone de una parte en la cual se explica el proceso para sumar y restar polinomios, una segunda parte donde se propone una actividad donde suman y restan por medio de la caja; esta actividad busca, en primera instancia, desarrollar habilidad operacional, en segunda instancia, inducir al estudiante a abstraer el método para sumar y restar polinomios de manera formal a partir de lo hecho, así como también de preguntas y respuestas.

\section{ANÁLISIS DE LA EXPERIENCIA}

La motivación principal que llevó a la planeación y ejecución de este trabajo, antes que restar mérito a una metodología de trabajo en particular, fue identificar cuantitativamente y cualitativamente los beneficios que ofrece cada una de ellas, a la hora de elegir una metodología para la enseñanza de las operaciones de suma y resta de polinomios, pues la metodología que se elija debe responder positivamente a múltiples variables, 
desde el punto de vista pedagógico, didáctico, organizacional, logístico. Por ello, se torna necesario realizar estudios que permitan hacer una caracterización de todas las posibles opciones que un docente tiene a la mano para enseñar, lo cual le permitirá elegir la que más se ajuste a las necesidades de un grupo en particular, siendo plenamente conscientes que cada grupo es muy diferente a los demás.

\section{CARACTERIZACIÓN DE LOS GRUPOS}

Los grupos que se eligieron para aplicar esta metodología de trabajo se conforman por hombres y mujeres, cuyas edades oscilan entre los 12 y 17 años, lo que indica que están en el rango de edad adecuado para cursar el grado octavo en una institución educativa formal. Ninguno de ellos tiene diagnóstico de problemas de aprendizaje o necesidades educativas especiales (NEE), así como tampoco discapacidades físicas que limiten el aprendizaje.

\section{Grupos EEE}

Los grupos 8.2 y 8.4 con quienes se trabajó la metodología EEE, se conforman por 43 y 46 estudiantes respectivamente, entre hombre y mujeres, cada uno.

\section{Grupos CP}

Los grupos 8.1 y 8.3 están compuestos por 43 y 40 estudiantes respectivamente, entre hombres y mujeres. No hay presencia de estudiantes repitentes, y como aspecto diferenciador es importante mencionar que son grupos en los que se facilita lograr concentrarlos y disponerlos para el trabajo. Un aspecto relevante de 8.3 es que la inasistencia ha sido un factor determinante que incide negativamente en el aprendizaje, pues el ausentismo por parte de varios estudiantes es muy alto. Por su parte, en 8.1, si bien hay inasistencia alta, se concentra en dos, máximo tres estudiantes, es decir, son esos tres estudiantes los que acumulan la mayor parte de inasistencias a las clases de matemáticas.

\section{ANÁLISIS CUANTITATIVO}

Para realizar el análisis cuantitativo de la eficiencia que tuvo cada uno de los dos métodos, se aplicaron dos pruebas distintas.
Solución de ejercicios: se planteó con el fin de verificar el nivel alcanzado por cada estudiante. Consistió en proponer 10 parejas de polinomios, con ellas plantear y resolver la suma y resta. Los polinomios propuestos van aumentando en nivel de complejidad, pues se inicia con polinomios cuyos términos tienen coeficiente entero y la parte literal compuesta por una sola letra, coeficientes fraccionarios, parte literal compuesta por dos o más letras con coeficientes enteros, y finalmente, términos con coeficientes fraccionarios y dos o más letras en la parte literal y exponentes literales. La escala evaluativa es la escala oficial de la institución, esto es, de 0 a 5 puntos. A cada ejercicio se le asignó igual valor numérico en cuanto a nota, siendo de 0.5 por ejercicio.

Exposición: con el fin de establecer si los estudiantes incorporaron en su estructura cognitiva el concepto de suma y resta de polinomios, se asignó a cada estudiante una pareja de polinomios, con el objetivo de exponer el proceso de suma y resta, en entrevista privada con el docente, argumentando cada paso que aplicó. Este proceso se evaluó como correcto si el estudiante explicó acertadamente suma y resta, incorrecto en cualquier otro caso.

Las siguientes tablas con sus respectivos gráficos muestran los resultados obtenidos por cada grupo, en cada una de las actividades evaluativas.

\section{Solución de Ejercicios}

Tabla 1: Cantidad de ejercicios bien resueltos por grupo

\begin{tabular}{ccccc} 
Rango & 8.1 & 8.2 & 8.3 & 8.4 \\
\hline 0 a 5 & 9 & 14 & 10 & 14 \\
6 a 7 & 23 & 12 & 21 & 20 \\
8 a 9 & 8 & 12 & 4 & 8 \\
10 & 3 & 5 & 5 & 4 \\
Total & 43 & 43 & 40 & 46 \\
\hline
\end{tabular}


Figura 9: Diagrama de barras ejercicios bien resueltos por grupo.

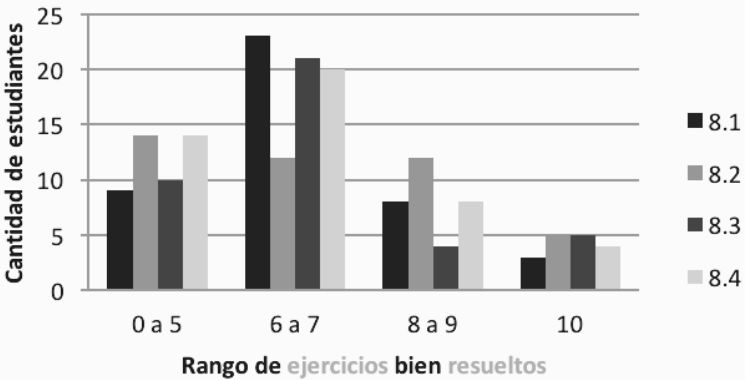

Tabla 2: Promedios y desviaciones estándar por grupo y metodología

\section{$\begin{array}{llllll}8.1 & 8.2 & 8.3 & 8.4 & C P & E E E\end{array}$}

Promedio $\quad 3,2 \quad 3,0 \quad 3,0 \quad 2,9 \quad 3,1 \quad 2,9$

Estándar $\quad 1,1 \quad 1,6 \quad 1,2 \quad 1,4 \quad 1,2 \quad 1,5$

De la tabla 2 se observa que los promedios por grupos y por metodologías de trabajo no presentan dispersión muy significativia, lo cual implica que la efectividad de la metodología alternativa no se centra en este aspecto. Pero las desviaciones estándar de cada grupo y de cada metodología sí llevan a concluir que con la metodología alternativa se logra que la mayor parte de los estudiantes se acerquen, en cuanto a notas se refiere, a la media del grupo; por su parte, la metodología tradicional genera mayor dispersión de notas, lo que significa que hay estudiantes que alcanzan notas muy altas, al tiempo que hay otra parte de los estudiantes con notas muy bajas.

\section{Exposición}

Tabla 3: Sustentaciones por grupo

\begin{tabular}{ccccc} 
Nota & 8.1 & 8.2 & 8.3 & 8.4 \\
Correcto & 25 & 14 & 16 & 22 \\
Incorrecto & 18 & 29 & 24 & 24 \\
Total & 43 & 43 & 40 & 46 \\
\hline
\end{tabular}

Figura 10: Diagrama de barras resultados exposición, por grupo.

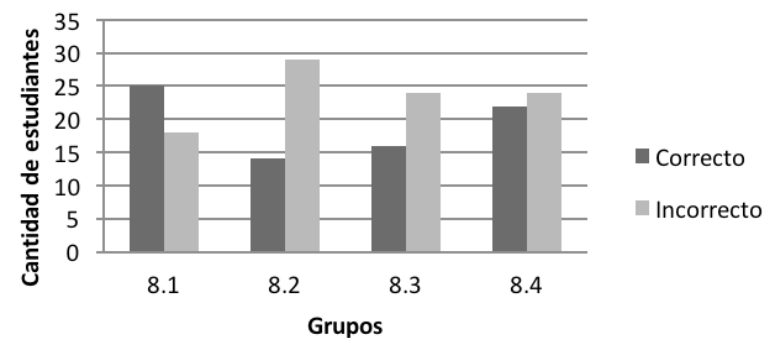

\section{ANÁLISIS CUALITATIVO}

El siguiente análisis, antes que ser un compilado de argumentos de diferentes autores, surge de la reflexión pedagógica y didáctica que se hizo después de haber finalizado la experiencia. Los años de servicio, los diferentes grupos a quienes se ha servido, el haber ofrecido en reiteradas ocasiones y de manera continua el curso de álgebra de grado octavo, el conocimiento que se ha ido adquiriendo, voluntaria o involuntariamente, entorno a los patrones de comportamiento de los estudiantes, permiten que los maestros identifiquen las características que debe tener una metodología de trabajo en cada uno de los grupos con los que se trabaja. Es innegable el hecho de que si determinada metodología surtió efecto positivo con un grupo, no es garantía de que surta el mismo efecto con los demás. En consideración de lo expuesto, surge la necesidad de analizar características de la experiencia que quedan excluidas del análisis cuantitativo, pues hay muchos aspectos de alta relevancia dentro del aula de clase que los números no alcanzan a percibir.

La intención de este análisis no es restar valor a una metodología u otra, por el contrario, es tratar de buscar aspectos que ayuden y que aporten positivamente a la hora de elegir una metodología de trabajo para enseñar suma y resta de polinomios, sin perjuicio de que puedan ser adaptadas y aplicadas en otras temáticas. Es por ello que se plantea un paralelo entre las dos metodologías, tratando de mostrar las fortalezas de cada una, en aspectos que consideramos básicos y fundamentales que se debe tener en cuenta cuando se quiere elegir un método para enseñar. 
TIEMPO

Es fácilmente manipulable, pues dependiendo del grado de complejidad al que se quiera llegar, se puede predeterminar y discriminar con anticipación.

\section{MOTIVACIÓN}

ORGANIZACIÓN

\section{INTERACCIÓN DOCENTE \\ - ESTUDIANTE}

\section{RECURSOS}

\section{SATISFACCIÓN}

\section{INTERACCIÓN ESTUDIANTE - ESTUDIANTE}

Se evidencian niveles muy bajos, con excepción de los estudiantes que sienten agrado por el trabajo en matemáticas.

Con este método se torna más sencillo controlar la organización de los grupos.

Es mucho más frecuente en la etapa de explicación, pues es en ésta donde se abre el espacio para las dudas. Hay picos de interacción cuando el estudiante se enfrenta a los primeros ejercicios.

En esta investigación se hizo el esfuerzo para entregar a cada estudiante el paquete completo de ejercicios que se trabajarían, con el fin de optimizar el tiempo.

Desde el punto de vista del aprendizaje, la mayoría de los estudiantes manifestaron estar satisfechos porque aprendieron e interiorizaron la técnica para sumar y restar polinomios.

Desde el punto de vista del goce y disfrute que produjo el trabajo con esta metodología, se encuentra que, como era de esperarse, la gran mayoría manifiesta que el proceso no generó agrado porque "todo parece parte de un libreto", se explica, se muestran ejemplos, $\mathrm{y}$ se proponen muchos ejercicios para afianzar lo que se entendió de la explicación. "Es muy aburrido”, argumentan los estudiantes.

Dado que se entregó material para cada estudiante, la interacción entre pares realmente fue mínima, además de que el docente sugirió que todas las preguntas que pudieran surgir después de la etapa de explicación se las realizaran directamente a él, con el fin de ser preciso en la solución de la inquietud.
El tiempo está predeterminado, pero requiere destinar sesiones adicionales para explicar el manejo de la caja de polinomios.

El hecho de trabajar con material diferente a papel y lápiz genera expectativas. Además, al estar fundamentada en un juego tradicional (rompecabezas), genera sensaciones de alegría.

El orden inicial de la clase se ve sumamente alterado, principalmente en las primeras sesiones de trabajo.

En ciertos momentos el proceso de interacción docente-estudiante se torna frecuente, sobre todo cuando se inicia el trabajo en fundamentación, y en la última parte de la guía, que es en donde el estudiante se enfrenta al proceso de abstracción; ocurre de igual manera cuando se enfrenta a los ejercicios para verificar si efectivamente alcanzó el objetivo del proceso.

Tablas de MDF, cartón paja, guillotina, tijeras, vinilos, marcadores.

Fotocopias de las guías de aprendizaje, fotocopias de los ejercicios de verificación.

Desde el punto de vista del aprendizaje efectivo, los estudiantes manifiestan que es realmente complicado establecer una correlación entre los fundamentos de la caja de polinomios y los diferentes tipos de polinomios que se les puedan presentar para ser sumados o restados, es decir, llevar a cabo el proceso de abstracción es muy complicado.

Los estudiantes manifiestan que la experiencia de trabajar con la caja de polinomios fue agradable, porque nunca se llegaron a imaginar que temas del nivel de abstracción como lo son la suma y resta de polinomios pudiesen ser abordados con una herramienta que se fundamenta en un juego de disposición de rectángulos y cuadrados de varios colores.

A pesar de haber construido material para cada estudiante, se esperaba y efectivamente sucedió, que los estudiantes interactuaron mucho entre pares por la emoción que genera el hecho de sentir que antes que estar aprendiendo están jugando. El juego es una actividad de tipo social, y dado que la herramienta se fundamenta en él, era de esperarse que el índice de interacción fuese muy alto. didácticas para

la enseñanza

de la suma

y la resta de
| Panorama 


\section{CONCLUSIONES}

1. El material didáctico actúa como facilitador para generar mayor interacción e intercambio de saberes dentro del aula de clase. En comparación con la metodología tradicional, la caja de polinomios supera ampliamente los niveles de interacción entre docente-estudiante y estudiante-estudiante.

2. El trabajo con la caja de polinomios, bajo los criterios ausubelianos, facilita el planteamiento y formulación de preguntas por parte de los estudiantes, porque la nueva información está asociada a conceptos ya incorporados a la estructura cognitiva, lo cual implica que el estudiante no debe realizar procesos de abstracción de alto nivel para poder preguntar, pues todo está asociado a lo que ya sabe.

3. Utilizando la caja de polinomios se logra reducir la dispersión de las notas de los estudiantes, lo que significaría que la gran mayoría alcanzaría el mismo nivel de apropiación de la nueva información. Con el método tradicional, el promedio de notas es muy similar al que se obtiene con la caja de polinomios, pero el índice de dispersión es mucho más elevado, es decir, algunos estudiantes obtienen notas muy altas, pero en contraste, algunos obtienen notas muy bajas, lo que llevaría a pensar que mientras algunos aprenden muy bien a sumar y restar polinomios, otra parte del grupo presenta muchas dificultades para aprender.

4. El proceso de abstracción es sumamente complejo, pues requiere de planeación detallada para lograr que los estudiantes lo alcancen con éxito. No se enseña a abstraer, pero por medio de la formulación de preguntas es posible inducir a efectuar procesos mentales propios del pensamiento matemático para abstraer propiedades, procesos, entre otros, de los objetos tangibles que se utilizan en el aula, lo que finalmente converge en el aprendizaje de los conceptos que se pretenden enseñar.

5. Enseñar con metodología tradicional puede lograr alcanzar niveles muy altos de apropiación de la nueva información, pues en todo momento del proceso se trabaja de manera abstracta con los objetos propios del tema, pero requiere que quien aprende tenga un alto nivel de manejo de subsumidores propios de cada nuevo tema, pues finalmente son ellos los que ligan la nueva información con la que ya se tiene en la estructura cognitiva.

\section{REFERENCIAS}

1. Agudelo, O., Ortiz C., Valbuena S. (2015). Desarrollo y evaluación de un material didáctico multimedia para facilitar el aprendizaje de las matemáticas. Revista Facultad de Ciencias Básicas. Universidad Nueva Granada, 11(1), 70-83. DOI: https://doi.org/10.18359/rfcb.382.

2. Gómez, C., Mosquera S., Soto F. (2005). La caja de polinomios. Revista de Matemáticas Escuela Regional de Matemáticas, 13(1), 83-97.

3. Lozano J., Naranjo C., Soto F. (2009). Aprendizaje de álgebra en grupos con discapacidad auditiva utilizando la caja de polinomios. Revista Sigma 9(1), 38-60.

4. Mallat, J. (2011). Didáctica: Concepto, objeto y finalidad. En N. Rajadell, y F. Sepúlveda. (Ed), Didácica General para Psicopedagogos, 1-31, España.

5. Moreira, M. (1997). Aprendizaje significativo: un concepto subyacente. Actas del Encuentro Internacional sobre el Aprendizaje Significativo, 19, 44.

6. Urbano, M. (2011). Experiencias docentes. Experiencia didáctica lúdica basada en el computador para enseñanza de polinomios en segundo año de educación básica. Pensamiento Matemático, 1(1), 1-21.

7. Villarroel,J. (2014). Propuesta para la enseñanza de las operaciones básicas (adición, sustracción, multiplicación y división) y el proceso de factorización de polinomios, con la herramienta didáctica la "caja de polinomios", en estudiantes de grado octavo de la I.E María Cano de Medellin (tesis de maestría). Universidad Nacional de Colombia, Medellín, Colombia.

8. Vinholi, J. (2011). Contribuições da Teoria da Aprendizagem Significativa para a aprendizagem de conceitos em Botânica. Acta Scientiarum. Education, 33(2), 281-288. DOI: 10.4025/actascieduc.v33i2.14355. 